\title{
REVIEW
}

\section{Are the therapeutic strategies in anorexia of ageing effective on nutritional status? A systematic review with meta-analysis}

\author{
S. Perna, ${ }^{1}$ (D) M. Rondanelli, ${ }^{1,2}$ D. Spadaccini, ${ }^{1}$ A. Lenzi, ${ }^{3}$ L. M. Donini ${ }^{3}$ \& E. Poggiogalle ${ }^{3}$ \\ ${ }^{1}$ Department of Public Health, Experimental and Forensic Medicine, Section of Human Nutrition, Endocrinology and Nutrition Unit, Azienda di \\ Servizi alla Persona, University of Pavia, Pavia, Italy \\ ${ }^{2}$ IRCCS Mondino Foundation, Pavia, Department of Public Health, Experimental and Forensic Medicine, Unit of Human and Clinical Nutrition, \\ University of Pavia, Italy \\ ${ }^{3}$ Department of Experimental Medicine, Section of Medical Pathophysiology, Food Science and Endocrinology, Sapienza University of Rome, \\ Rome, Italy
}

\author{
Keywords \\ anorexia of ageing, body weight, elderly, \\ malnutrition, senile anorexia.

\section{Correspondence \\ S. Perna, Department of Public Health, Experimental and Forensic Medicine, Section of Human Nutrition and Dietetics, Azienda di Servizi alla Persona di Pavia, University of Pavia, Via Emilia 12, Pavia, Italy. \\ Tel.: 0039-0382381749 \\ Fax: 0039-0382381218 \\ E-mail: simoneperna@hotmail.it}

\section{How to cite this article}

Perna S., Rondanelli M., Spadaccini D., Lenzi A., Donini L. M. \& Poggiogalle E. (2018) Are the therapeutic strategies in anorexia of ageing effective on nutritional status? A systematic review with meta-analysis. J Hum Nutr Diet. https://doi.org/10.1111/jhn.12594

\begin{abstract}
Background: Anorexia of ageing (AA) may be considered as a risk factor for frailty and has an important impact on quality of life, morbidity and mortality. Methods: A systematic review and a meta-analysis were performed to summarise the results from several trials on the effectiveness of treatments in $\mathrm{AA}$, as associated with depression, sensory impairment of taste and smell, decreased appetite or early satiety, and disability. Eligible studies were required to report baseline and follow-up values, the mean change $(\Delta-$ change) from baseline, and/or the mean difference among intervention groups versus control group, concerning food intake (kcal/daily) and/or nutritional outcomes, such as body weight, body mass index, albumin and Mini Nutritional Assessment.

Results: The systematic review included 20 papers based on different therapeutic approaches concerning food intake and/or nutritional outcomes. The results of the meta-analysis indicate that the interventions for AA have an important impact on body weight $[+1.59 \mathrm{~kg} ; 95 \%$ confidence interval $(\mathrm{CI})=1.48-+1.71 \mathrm{~kg} ; P<0.001)$ and on energy intake $(+56.09 \mathrm{kcal} ; 95 \%$ $\mathrm{CI}=-54.05$ to $+166.25 \mathrm{kcal} ; P=0.32)$. Regarding secondary outcomes, it was not possible to meta-analyse the limited amount of data availab le.

Conclusions: The different variants of AA need to be defined because diverse therapeutic approaches are available. A more precise definition of the functional impairments associated with AA may allow a more correct decision about the most appropriate therapy to be prescribed. Moreover, this may allow for a more effective performance of the different therapeutic approaches once they are better targeted to the different scenarios of AA.
\end{abstract}

\section{Introduction}

A reduction of appetite and food intake, namely, anorexia of ageing (AA), is frequently observed in the elderly; it can be considered as a risk factor for frailty, and it has a relevant impact on quality of life, morbidity and mortality ${ }^{(1-6)}$. Indeed, several studies conducted in communitydwelling and institutionalised elderly subjects have indicated that AA and unintentional weight loss are dominant risk factors for protein-energy malnutrition, sarcopenia, physical frailty and mortality, independent of age, sex and other potential confounders ${ }^{(7,8)}$. A number of screening tools are available to identify subjects at risk of developing anorexia, including Functional Assessment of Anorexia/Cachexia Therapy; the Appetite, Hunger and Sensory Perception Questionnaire; and the Simplified 
Nutritional Assessment Questionnaire ${ }^{(9-12)}$. In clinical practice, AA can be diagnosed when, in the absence of alterations in the oral cavity that can affect the physiological act of eating and/or masticatory function, spontaneous food intake is significantly reduced $(\leq 50 \%$ of the standard portion served or $\geq 25 \%$ of food left uneaten at two-thirds of meals for $\geq 3$ days) ${ }^{(13-15)}$.

The prevalence of AA is highly variable in the different epidemiological studies; it is approximately 30\% in hospitalised subjects in acute care and rehabilitation wards and it decreases in free-living subjects; however, it remains at approximately $10 \%^{(7,16,17,18)}$. Anorexia should not be considered as an inevitable 'side effect' of ageing because many risk factors, such as disability, depression, sensory impairments, reduced chewing efficiency and a decline in gastrointestinal functions, can be identified and potentially corrected ${ }^{(19)}$.

Macronutrient distribution can affect oral intake; a higher proportion of energy from fat appears to increase the satiety signal from glucagon-like peptide-1 and decrease hunger ${ }^{(20)}$. Protein intake moderately beyond the recommended dietary allowances in older adults appears to have a beneficial effect on muscle protein anabolism and appetite regulation, favouring the preservation of muscle mass at the same time as controlling body fat (20). Different studies have confirmed the positive effects of oral nutritional supplements in malnourished older adults and different pharmaceutical approaches have been used in AA (e.g. megesterol, meclobemide, tetrahydrocannabinol, cyproheptadine, cholecystokinin antagonists), although the results are conflicting ${ }^{(21-25)}$. Improving the ambiance of dining rooms and assistance with meals (i.e. allowing adequate time for caregivers to feed subjects with disability or 'slow-eaters'), encouraging the relatives to be present during the mealtime and providing them with ethnically appropriate food choices may be helpful with respect to improving the nutritional status of freeliving elderly subjects and nursing home residents ${ }^{(26-28)}$.

Therefore, a systematic review and a meta-analysis were performed to summarise the findings from several trials on the effectiveness of treatments in AA.

\section{Materials and methods}

The present systematic review was conducted in accordance with the PRISMA (Preferred Reporting Items for Systematic Review and Meta-Analyses) statement ${ }^{(29)}$.

It was performed using the steps:

- Formulation of the review question: 'anorexia of ageing: treatment procedures';

- Definition of participants: geriatric patients ( $\geq 65$ years), both sexes, from all settings;
- Search strategy for the identification of relevant intervention studies on anorexia of ageing;

- Analysis of the data through the systematic review and meta-analysis.

\section{Search strategy}

English-written articles were identified by searching the Medline database ${ }^{(30)}$, Scopus ${ }^{(31)}$, ISI Web of Science ${ }^{(32)}$ and Google Scholar ${ }^{(33)}$.

The search strategy was based on the search terms: 'anorexia of aging' AND 'sensory impairment' OR 'taste impairment' OR 'smell impairment' OR 'swallowing impairment' OR 'chewing impairment' OR 'depression' OR 'feeding' OR 'oral supplement' OR 'enriched meal OR' exercise' OR 'nutritional intervention'. In a first stage, four individuals (SP, MR, LMD and EP) independently performed a systematic review of the literature, employing the same search strategy. In a second stage, duplicate records were removed and discrepant records were carefully investigated within these four individuals before selecting retrieved papers for full-text review.

Eligible studies were required to report baseline and follow-up values, the mean change ( $\Delta$-change) from baseline, and/or the mean difference among intervention groups versus control group, concerning food intake ( $\mathrm{kcal} \mathrm{day}^{-1}$ ) and/or nutritional outcomes [such as body weight (BW), body mass index (BMI), plasma albumin levels, Mini Nutritional Assessment (MNA) score]. Studies in subjects with any cancers in the terminal phase, with good nutritional status $(\mathrm{MNA}>23.5)$, with any acute diseases, uncertain life expectancy or undergoing artificial nutrition, palliative care or chemotherapy, were excluded.

\section{Analysis of the data and presentation of the outcomes}

Clinical trials investigating the effectiveness of dietary or pharmacological treatment in AA were included. For each study, the data specified were: the author, the name of the journal in which the study was published and the year of publication, the number and age of participants enrolled in the study, the drop-out rate, the study characteristics, and the therapeutic intervention, duration and outcomes. A meta-analysis for pooled estimate for aggregated data was performed.

\section{Risk of bias in individual studies}

The risk of bias of each study was assessed using the Cochrane Collaboration Risk of Bias tool ${ }^{(34)}$ and, considering as factors contributing to the study quality, the generation of the allocation sequence, allocation concealment, blinding of outcome data, the presence of incomplete data and selective reporting. These factors were 
classified as low risk of bias, high risk of bias or an unclear risk of bias. Studies with a low risk of bias for at least three items were considered as good; studies with a low risk of bias for at least two items were considered as fair, and studies with a low risk for no item or only for one item were regarded as poor. Only randomised controlled trials were included in the meta-analysis.

\section{Results}

The literature search retrieved 322 papers via database searching and 84 papers were selected via full-text revision. Sixty-four studies were excluded. The 20 remaining studies (17 clinical trials, all presenting a control group and three observational studies) were selected for the present systematic review. The systematic review includes 20 studies with a total of 1515 adults aged 59-99 years. Of these 20, 12 randomised clinical trials were included in a meta-analysis. Figure 1 shows the study selection procedure.

The general data concerning the different studies are provided in the Supporting information (Table S1), as are the specific interventions and the results obtained (see Supporting information, Table S2). The duration of the studies was from 8 weeks to 24 months.

The inclusion criteria considered in the selected studies are very different, with a diverse combination of parameters: accidental weight loss, low BMI $(<18,21$ or $25 \mathrm{~kg} \mathrm{~m}^{-2}$ in different studies), BW below $80 \%$ of ideal weight, reduced levels of albumin ( $<35$ or $38 \mathrm{~g} \mathrm{~L}^{-1}$ in different studies), prealbumin serum levels $<0.18 \mathrm{~g} \mathrm{~L}^{-1}$, MNA score below 23.5, the concurrence of acute illness or the presence of chronic illness or dementia, and the need for assistance during meals.

The definition of AA in the different studies was based on:

- Reduced dietary intake [not specifically quantified in six studies; objectively quantified (left more than 25\% of their food uneaten at two-thirds of meals) in two studies; evaluating reduced dietary intake and sensory perceptions (olfactory sensitivity; appetite, hunger, sensory perception) in one study];

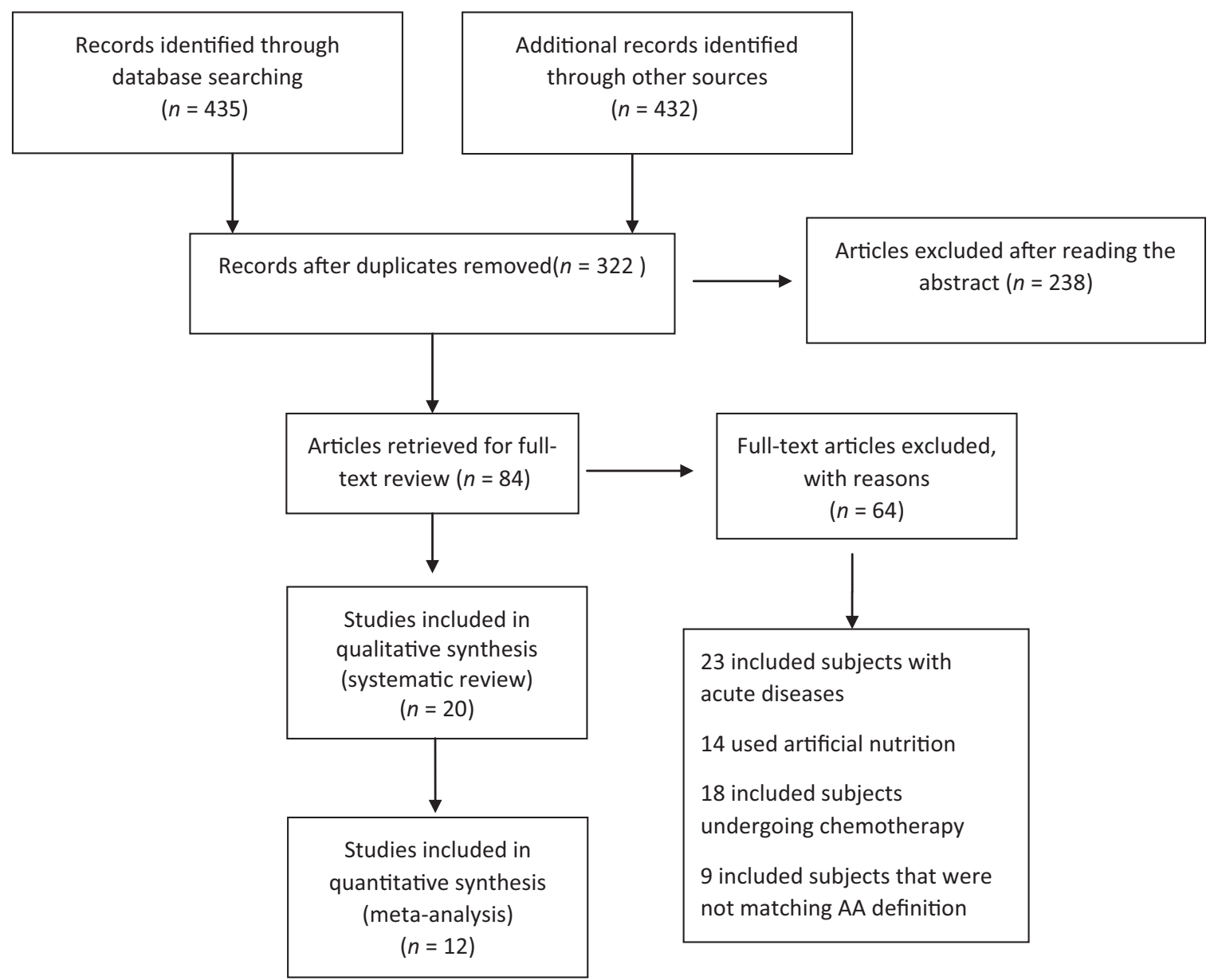

Figure 1 Flow diagram of the study. 
- Self-reported poor or fair appetite in one study;

- MNA score (considering the item aimed at assessing appetite in one study or the global score referring to the definition of 'at risk of malnutrition' in four studies);

- The presence of malnutrition (one study) or risk of undernutrition according to the physician judgment (two studies);

- The presence of disability that can affect eating in three studies.

The types of intervention were classified into five groups: pharmacological approaches $(13,35,36)$, nutritional interventions or supplementation ${ }^{(37-46)}$, flavour enhancement ${ }^{(47-49)}$, assistance with meals ${ }^{(50-52)}$, combination of nutritional intervention and exercise programme ${ }^{(53)}$.

\section{Results of interventions}

\section{Considering the different interventions}

- Pharmacological approaches: antidepressants were effective with respect to increasing BW, MNA score and serum albumin levels significantly $(P<0.05)$ in subjects without dementia ${ }^{(35)}$, whereas, in the study conducted by Wilson et al. (13) (using dronabinol), weight gain was not significant. Megestrol acetate was used in one randomised controlled trial conducted in 47 elderly subjects, recently discharged from an acute care hospital, with fair or poor appetite. Participants were randomised to four different intervention groups (placebo or megestrol acetate $200,400 \mathrm{mg}$ or $800 \mathrm{mg} \mathrm{day}^{-1}$ ) for 9 weeks. No significant differences were found between treatment groups with respect to appetite and albumin levels, whereas prealbumin increased in a dose-response relationship ${ }^{(36)}$.

- Nutritional intervention or supplementation: hot evening meal, protein and energy enriched soups and sauces or supplementation [with cookies or oral nutritional supplements (ONS)] were effective with respect to improving significantly $(P<0.05)$ nutritional parameters (energy intake, BW, BMI, albumin concentrations, MNA score) $(37,39-43,45)$. ONS were not effective only in the study conducted by Bos et al. ${ }^{(44)}$, whereas a hot evening meal was not effective in the study conducted by Odlund et al. ${ }^{(38)}$;

- Flavour enhancement: flavour enhancers and sauces added to the main dish were effective with respect to significantly increasing BW or energy intake in two studies $(47,49)$, whereas they did not improve any nutritional parameters in the study by Essed et al. ${ }^{(48)}$;

- Meal assistance: assistance with the meal was effective with respect to significantly increasing different nutritional parameters especially energy intake and MNA score $(P<0.05)$, whereas it did not affect BW or BMI ${ }^{(50-52)}$;

- Combination of nutritional intervention and exercise: in the only study adopting this approach, this was not found to be effective with respect to improving BW, BMI, energy intake or albumin concentrations ${ }^{(53)}$.

\section{Considering the different nutritional outcomes:}

- Changes in body weight: seven clinical trials $(35,37,39,40,45-47)$ using different approaches, including antidepressants, hot evening meal, protein and energy enriched soups and sauces supplementation with cookies, flavour enhancers and ONS, demonstrated a statistically significant increase in intervention groups. BW varied from +0.73 to $+2.4 \mathrm{~kg}$ depending on the study $(P<0.05)$. Five other trials using different approaches, such as assistance with the meal, flavour enhancers and other nutritional interventions or supplementation, found no significant weight gain ${ }^{(44)}$ or registered a nonstatistically significant weight gain in the intervention groups compared to the control groups $(38,42,43,50)$. Finally, two observational studies reported contradictory observations: in the study conducted by Wilson et al. ${ }^{(13)}$ (using dronabinol), the increase in BW was not significant, whereas, in the study by Cruz-Jentoft et al. ${ }^{(43)}$, the use of ONS led to a significant increase in BW $(+2.1 \mathrm{~kg} ; P<0.0001)$;

Changes in body mass index: in three randomised clinical trials based on nutritional interventions or supplementation (39,40,45), BMI increased significantly in intervention groups (BMI: $+0.5,+0.8$ and $+1.3 \mathrm{~kg} / \mathrm{m}^{2}$, respectively; $P<0.05$ ) compared to the control group, whereas the change in BMI was not significant in other studies using hot evening meals, feeding assistance or ONS ${ }^{(38,43,51)}$;

- Changes in albumin levels (in $g L^{-1}$ and/or as a percentage): the three studies that evaluated the effects of interventions in terms of albumin levels produced contrasting results: both significant and nonsignificant increases or decreases. In a longitudinal study using antidepressants ${ }^{(35)}$ albumin levels increased significantly $\left(+1.78 \mathrm{~g} \mathrm{~L}^{-1} ; \quad P<0.05\right)$ in subjects without dementia, whereas the increase in subjects with dementia was not statistically significant. In another study using ONS ${ }^{(39)}$, a statistically significant decrease in albumin levels was observed in both intervention and control groups $(-2.0$ and $-2.6 \%$, respectively; $P<0.05$ ). In a randomised clinical trial performed by Wouters-Wessling et al. ${ }^{(40)}$ (using ONS), a nonsignificant increase in albumin levels was found in both intervention $\left(+1.3 \mathrm{~g} \mathrm{~L}^{-1}\right)$ and control groups $\left(+1.4 \mathrm{~g} \mathrm{~L}^{-1}\right)$;

- Changes in Mini Nutritional Assessment score: three randomised controlled trials ${ }^{(42,45,50)}$ and one observational study (43) using nutritional intervention/supplementation and assistance with meal showed a significant increase in MNA score in intervention groups $(+3.6,+3$, +1 and +2.5 points, respectively; $P<0.05$ ). The results of the study conducted by Thomas et al. (35) (based on 
antidepressant therapy) showed a significant increase in MNA score $(+0.76$ points; $P<0.05)$ in subjects without dementia, whereas, in subjects with dementia, the increase was not significant;

- Changes in energy intake: four studies ${ }^{(41,49,50,52)}$ (using different approaches: dronabinol, nutritional intervention or supplementation, assistance with a meal) showed a statistically significant increase in energy intake in intervention groups (energy intake variation: from +115 to +341 kcal day; $P<0.05)$. By contrast, two studies $(48,53)$ (using assistance with a meal and a combination of nutritional intervention and exercise programme, respectively), found no significant variation in energy intake in the intervention groups.

\section{Meta-analysed data}

The meta-analysed mean differences for random effects (MD) showed a significantly greater increase in BW $(\mathrm{MD}=-1.59 \mathrm{~kg}$; range $=1.47$ to $1.71 ; P<0.01)$ (Fig. 2$)$ and in energy intake $(\mathrm{MD}=56.09 \mathrm{kcal}$; range $=-54.05$ to $166.24 ; P=0.32)$ regardless of the approach adopted to treat AA (Fig. 3).

In the five studies $(40,42,47,48,50)$ (242 subjects in the intervention groups and 232 subjects in the control groups) that considered $\mathrm{BW}$ as an outcome variable, there was a significant increase (Fig. 2) when comparing the results of the intervention groups with those of the control groups. The test for heterogeneity indicated that the treatment effect was not significantly different between the considered studies $(P<0.93)$ with $I^{2}$ values $=0 \%$. Mean differences of random effects showed a change in BW of $-0.8(-7.68,+6.08) \mathrm{kg},-0.0(-6.8$,
$+6.8) \mathrm{kg},-0.90(-7.75,+5.95) \mathrm{kg}{ }^{(48)},+1.51(+0.09$, $+2.93) \mathrm{kg}{ }^{(42)},+1.40(+0.69,+2.11) \mathrm{kg}^{(47)},+1.60(+1.48$, $+1.72) \mathrm{kg}^{(50)},+2.2(+0.41,+3.99) \mathrm{kg}{ }^{(40)}$ between groups, as shown in Fig. 2 as mean difference IV random effects.

In four studies ${ }^{(41,48,50,53)}$ considering the energy intake as the outcome variable (634 subjects in the intervention groups and 265 subjects in the control groups), there was no significant increase in energy intake (Fig. 3) when comparing the results of the intervention groups to the control groups. However, the tests for heterogeneity indicated that the treatment effect was significantly different between the different studies $(P<0.001)$ with $I^{2}=96 \%$. Mean differences of random effects between groups showed changes in energy intake of $+36(-67,+139){ }^{(41)}$, $0(-171,+171),+141(-52,+335),+119(-75,+314)$ $(53),-20(-85,+45),-16(-70,+37),-5(-61,+51)$ (48) and $+215(+202,+228){ }^{(50)} \mathrm{kcal}$, as shown in Fig. 3 as mean difference IV random effects.

\section{Risk of bias}

Figure 4 shows the methodological quality of the studies. Only nine studies were suitable for all the five items considered for the methodological quality assessment (six studies were blinded for the variables considered). In summary, no studies were rated as appropriate in random sequence generation and in allocation concealment, $75 \%$ of the studies were rated as appropriate in blinding of participants and personnel, 75\% of the studies were adequate in blinding of the outcome assessment, $80 \%$ reported incomplete outcome data, 90\% gave only a selective reporting, and $75 \%$ showed other bias.

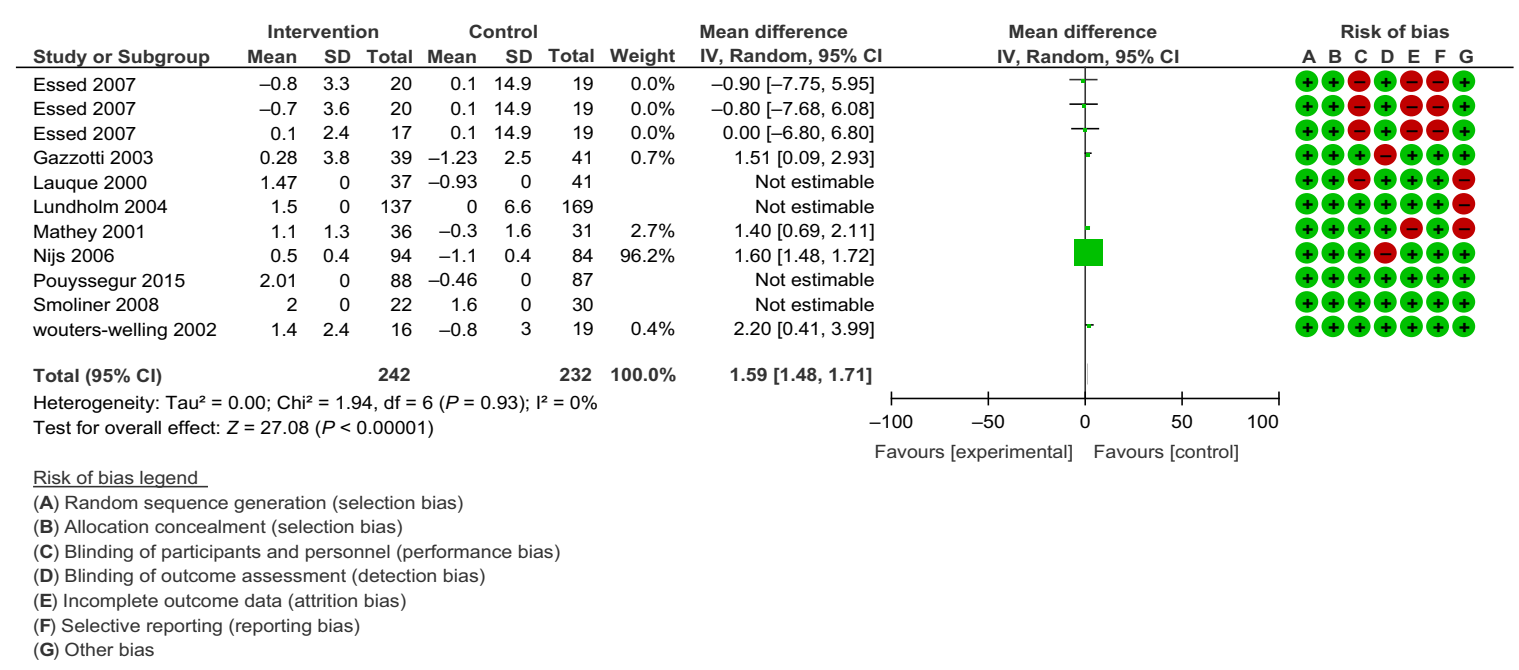

Figure 2 Effectiveness of treatments on body weight in the randomised controlled clinical trials included in the meta-analysis. $\mathrm{Cl}$, confidence interval. 


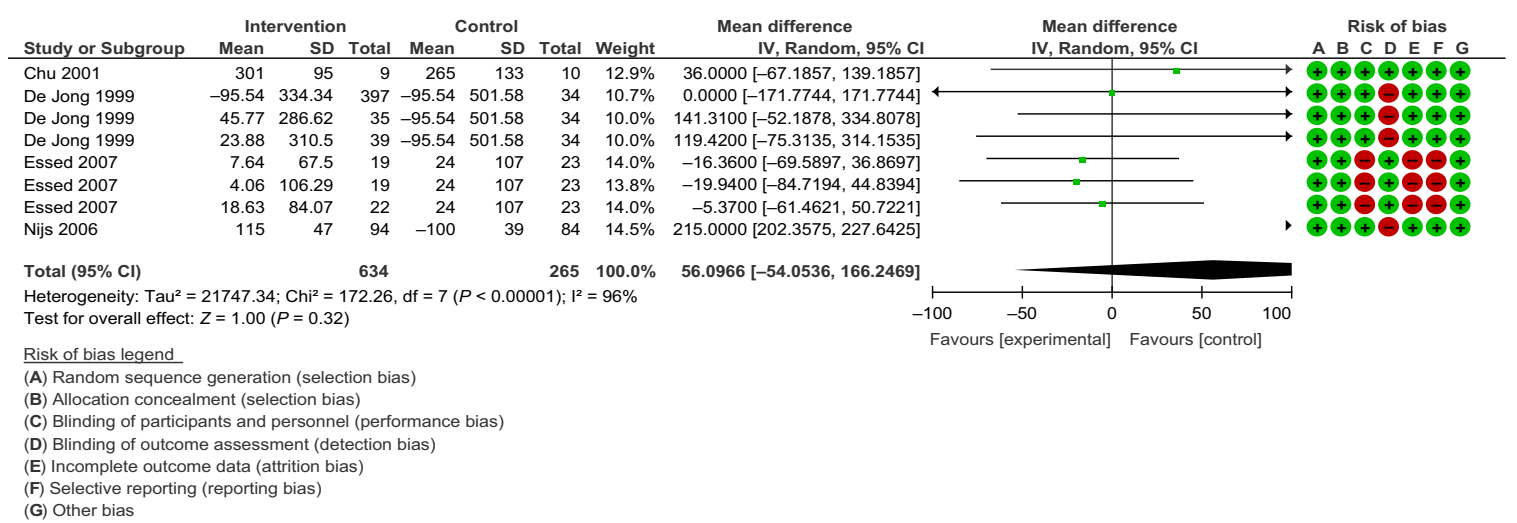

Figure 3 Effectiveness of treatments on energy intake in the randomised controlled clinical trials included in the meta-analysis. $\mathrm{Cl}$, confidence interval.

Random sequence generation (selection bias)

Allocation concealment (selection bias)

Blinding of participants and personnel (performance bias)

Blinding of outcome assessment (detection bias)

Incomplete outcome data (attrition bias)

Selective reporting (reporting bias)

Other bias

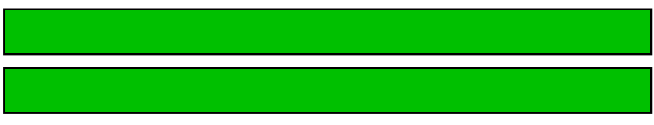

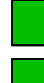
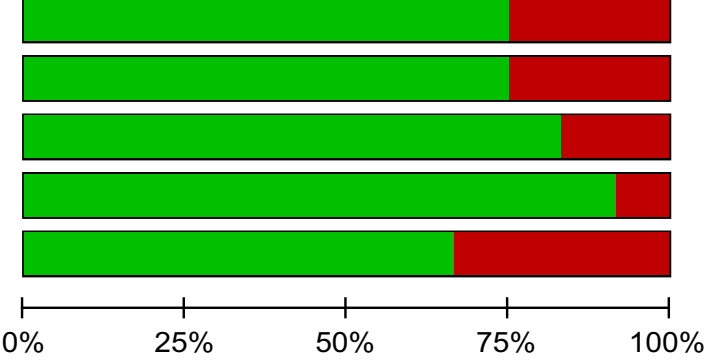

$\begin{array}{lllll} & 25 \% & 50 \% & 75 \% & 100 \%\end{array}$

\section{Low risk of bias}

Unclear risk of bias

High risk of bias

Figure 4 Summary of bias in either outcome considered: body weight and energy intake.

\section{Discussion}

This systematic review was able to select only a limited number of papers based on different therapeutic approaches, yielding contrasting results. The recent systematic review conducted by Malafarina et al. ${ }^{(54)}$ concluded that these treatments were not effective for AA because they were not able to modify significantly the concentration of peptides involved in appetite control.

The results of this meta-analysis indicate that the interventions for $\mathrm{AA}$ have an important impact on BW $(+1.59 \mathrm{~kg}, \quad P<0.001)$, whereas the effects on energy intake ( $+56.09 \mathrm{kcal}, P=0.32$ ) do not appear to be statistically significant. With regard to secondary outcomes, such as albumin levels, MNA score and BMI changes, it was not possible to meta-analyse the data as a result of the limited amount of data available.

Although numerous studies suggest different approaches for managing AA, evidence based on randomised controlled clinical trials is scarce. It is important to note that following a nutritional support could be difficult for this population as a result of the coexistence of many factors: loss of appetite, inactivity, sensory impairment, early satiation, psychological disorders and mental impairments. Furthermore, AA treatment requires a multidimensional approach, including food manipulation (improvement of flavour, food texture and palatability, increasing dietary variety, and feeding assistance), correction of environmental threat (preventing social isolation and improving conviviality, particularly in nursing home residents, providing adequate individual feeding assistance, changing the mealtime routine, modifying the dining environment, and staff training), reduction of pharmacological risk factors (a certain number of cardiovascular, psychiatric and anti-rheumatic drugs may reduce appetite) and treatment of underlying medical causes (swallowing disorders, dyspepsia, malabsorption syndromes, neurological causes, endocrine and psychiatric disorders, respiratory diseases and cardiovascular diseases), as recommended in mumerous studies ${ }^{(26,55-59)}$. 
Special attention should be also given to the nutritional screening tools, which constitute the primary intervention in the treatment of AA. MNA-Short Form and MNALong Form are considered among the best screening questionnaires, with a high accuracy for the prediction of malnourished elderly subjects, although the most recent versions of Nutritional Risk Screening also show good results in terms of compliance of subsequent nutritional treatments in younger residents of multiple healthcare sites, and perhaps this result could be significant also for older adults ${ }^{(60,61)}$. Additionally, the use of body composition and functional assessment techniques has become more frequent in the evaluation of nutritional status, with an improvement of the health outcomes of subjects, although clinical practice should evolve even more in this area, moving beyond current approaches ${ }^{(62)}$.

Pharmacological approaches to AA have focused on research trials, in particular on interventions that try to modify neuropeptide regulation (administration of ghrelin, cholecystokinin and Melanocortin 4 receptor antagonists, drugs blocking protein catabolism) ${ }^{(51)}$. Thomas et al. (35) examined the effects of antidepressant therapy (mainly serotonin reuptake inhibitors; SSRIs) in AA associated with depression. Antidepressants were effective with respect to increasing BW, MNA score and albumin concentrations both in subjects with or without dementia. Previously, a study by Hilas et al. ${ }^{(57)}$ involving mirtazapine assessed its favourable effects on appetite and weight in adults compared to placebo and other antidepressants. Mirtazapine may have multiple effects in subjects with weight loss because it relieves symptoms associated with depression, it regulates the gastrointestinal motor or sensory functions, and it significantly increases BW (mainly as a result of growth of visceral fat) ${ }^{(63)}$. Another study ${ }^{(64)}$, comparing the effects of older tricyclic antidepressants (TCAs) and newer SSRIs in relation to weight gain in nursing home residents showed that TCAs do not contribute to weight gain more than other antidepressants.

In studies that considered subjects with AA combined with decreased appetite ${ }^{(13,37-43)}$ or with early satiety $(44,45,53)$, interventions focused on nutritional interventions characterised by providing an additional intake of cookies with high protein/energy density, liquid oral supplements enriched with micronutrients, a hot evening meal or adding flavour enhancers to the main dish. The results obtained in most of these studies, where decreased appetite was involved, showed an increase in BW and energy intake, whereas ONS combined with an exercise programme was not effective in the studies where AA was combined with early satiety ${ }^{(65,66)}$. A recent systematic review of the literature suggests that increasing energy/nutrients density of food intake may increase energy intake in older adults with anorexia ${ }^{(67)}$. It should be emphasised, as suggested by Yeomans et al. ${ }^{(68)}$, that ONS preloads (mainly maltodextrin) may reduce the general desire to eat, whereas the administration of dietary supplements between meals is more effective with respect to increasing energy consumption ${ }^{(69)}$.

Sensory impairments in the elderly can lead to a reduced enjoyment of food and then to AA and, by this route, to a greater risk of frailty in community-dwelling elderly people ${ }^{(70)}$. A reduced ability to differentiate between various intensities or concentrations of taste and smell can result in anorexia ${ }^{(71)}$. With respect to the treatment of AA combined with sensory impairment of taste and smell, flavour enhancers or sauces added to the main dish were effective with respect to increasing BW or energy intakes in two studies ${ }^{(47,49)}$, whereas they did not elicit any improvements in nutritional parameters in the study by Essed et al. ${ }^{(48)}$. In the literature, considering different groups of subjects, the results remain contradictory: a study conducted by Havermans et al. ${ }^{(72)}$ found that flavour intensification did not alter sensory-specific satiety, whereas other studies suggest that the consumption of food with enhanced chemosensory properties could provide nutritional benefits and help to prevent weight loss. In particular, monosodium glutamate (MSG) appears to be effective with respect to improving both flavour enhancement and food intake ${ }^{(73)}$. However, in the study conducted by Essed et al. ${ }^{(48)}$ MSG $(0.5 \%$ and $2 \%$ ) did not appear to be effective with respect to improving food intake in an elderly sample. In addition, a recent systematic review considering studies conducted in all age groups could not provide sufficient evidence to recommend zinc supplementation for improving taste perception or acuity in zinc deficiency-related or idiopathic dysgeusia ${ }^{(74,75)}$.

The treatment of AA associated with disability is mainly characterised in the selected studies by assistance with meals and repeated measurement of BW (46,50-52). In this category of subjects, the administration of fruits and high protein supplements, as suggested by Lauque et al. (46), appears to be effective. Moreover, an exercise programme may affect the regulation of energy balance through the stimulation of corticotrophin-releasing hormone (CRH) and neuropeptide Y (NPY). In particular, NPY, by producing orexigenic effects and reducing thermogenesis, tends to counterbalance the physiological effects of $\mathrm{CRH}$ and therefore overcomes the effects of exercise on energy loss (76). Unfortunately, the only study considered in this systematic review using an exercise programme (focused on skill training, including strength, endurance, coordination and flexibility) (53) did not show beneficial effects: energy intake, BW and BMI were not positively affected by the intervention 
combining nutrient-dense products and an exercise programme.

\section{Strength and limitations of the present study}

The strength of this systematic review is represented by an attempt to analyse the practical interventions proposed for AA when considering diverse clinical scenarios based on its combination with different functional impairments. Indeed, AA combined with disability, for example, may be very different in clinical manifestations compared to AA combined with sensory impairment, depression or lack of appetite/early satiation. A limitation of the present study is therefore represented by the limited number of studies that were selected, or even available and appropriate for inclusion, especially for the meta-analysis that could consider only BW and energy intake as study outcome variables.

\section{Conclusions}

It is necessary to define the different variants of $\mathrm{AA}$ because diverse therapeutic approaches are available. A better definition of the functional impairments combined with AA may allow a more correct decision to be made about the most appropriate combination of therapies to be prescribed. Moreover, this may allow for a greater efficacy of the different therapeutic approaches once they are better targeted to the different clinical scenarios of AA. Finally, different treatments show a potentially favourable effect on BW (or BMI), energy intake, albumin levels and MNA score, suggesting the need for further research using long-term intervention studies in large study groups in with the aim of defining the best approach for the management of AA.

\section{Transparency declaration}

The lead author affirms that this manuscript is an honest, accurate and transparent account of the study being reported, that no important aspects of the study have been omitted and that any discrepancies from the study as planned have been explained. The reporting of this work is compliant with PRISMA (Preferred Reporting Items for Systematic Reviews and Meta-Analyses) guideline.

\section{Acknowledgments}

Everyone who contributed significantly to the work has been listed in the article and has provided their written consent.

\section{Conflict of interests, source of funding and authorship}

The authors declare that they have no conflict of interests.

No funding.

SP, MR, LMD and EP designed the study and search strategy, and also performed primary and secondary searches, title and abstract screening, full-text screening, analysis, quality assessment, and wrote the draft and final manuscript. DS provided consultation on the study design and data collection and critically reviewed the draft and final manuscript. AL performed the full-text screening and quality assessment, and also contributed to the final manuscript. SP and MR supervised the study design and critically reviewed and contributed to the final manuscript. EP and LMD supervised the study design and analysis and critically reviewed the manuscript. All authors critically reviewed the manuscript and approved the final version submitted for publication.

\section{References}

1. Morley JE (2017) Anorexia of ageing: a key component in the pathogenesis of both sarcopenia and cachexia. $J$ Cachexia Sarcopenia Muscle 8, 523-526.

2. Donini LM, Poggiogalle E, Piredda M et al. (2013) Anorexia and eating patterns in the elderly. PLoS ONE 8, e63539.

3. Landi F, Laviano A \& Cruz-Jentoft AJ (2010) The anorexia of aging: is it a geriatric syndrome? J Am Med Dir Assoc 11, 153-156.

4. Soenen S \& Chapman IM (2013) Body weight, anorexia, and undernutrition in older people. J Am Med Dir Assoc 14, 642-648.

5. Martone AM, Onder G, Vetrano DL et al. (2013) Anorexia of aging: a modifiable risk factor for frailty. Nutrients $\mathbf{5}$, 4126-4133.

6. Tsutsumimoto K, Doi T, Makizako H et al. (2017) The association between anorexia of aging and physical frailty: Results from the national center for geriatrics and gerontology's study of geriatric syndromes. Maturitas $\mathbf{9 7}$, 32-37.

7. Donini LM, Dominguez LJ, Barbagallo M et al. (2011) Senile anorexia in different geriatric settings in Italy. $\mathrm{J}$ Nutr Health Aging 15, 775-781.

8. Muscaritoli M, Anker SD, Argilés J et al. (2010) Consensus definition of sarcopenia, cachexia and pre-cachexia: joint document elaborated by Special Interest Groups (SIG) 'cachexia-anorexia in chronic wasting diseases' and 'nutrition in geriatrics'. Clin Nutr 29, 154-159. 
9. Wilson MMG, Thomas DR, Rubenstein LZ et al. (2005) Appetite assessment: simple appetite questionnaire predicts weight loss in communitydwelling adults and nursing home residents. Am J Clin Nutr 82, 1074-1081.

10. Ribaudo JM, Cella D, Hahn EA et al. (2000) Re-validation and shortening of the functional assessment of anorexia/ cachexia therapy (FAACT) questionnaire. Qual Life Res 9 , $1137-1146$.

11. Pilgrim AL, Baylis D, Jameson KA et al. (2016) Measuring appetite with the simplified nutritional appetite questionnaire identifies hospitalised older people at risk of worse health outcomes. J Nutr Health Aging 20, 3-7.

12. Savina C, Donini LM, Anzivino R et al. (2003) Administering the 'AHSP Questionnaire' (appetite, hunger, sensory perception) in a geriatric rehabilitation care. $J$ Nutr Health Aging 7, 385-389.

13. Wilson MM, Philpot C \& Morley JE (2007) Anorexia of aging in long term care: is dronabinol an effective appetite stimulant? - a pilot study. J Nutr Health Aging 11, 195198.

14. Donini LM, Savina C, Piredda M et al. (2008) Senile anorexia in acute-ward and rehabilitations settings. J Nutr Health Aging 12, 511-517.

15. Administration Health Care Financing (1999) Long Term Care Facility Resident Assessment Instrument (RAI) User's Manual, Minimum Data Set, Version 2. Natick, MA: Eliot Press.

16. Visvanathan R \& Chapman IM (2009) Undernutrition and anorexia in the older person. Gastroenterol Clin North Am 38, 393-409.

17. Landi F, Lattanzio F, Dell'Aquila G et al. (2013) Prevalence and potentially reversible factors associated with anorexia among older nursing home residents: results from the ULISSE project. J Am Med Dir Assoc 14, 119124.

18. Landi F, Russo A, Liperoti R et al. (2010) Anorexia, physical function, and incident disability among the frail elderly population: results from the ilSIRENTE study. $J$ Am Med Dir Assoc 11, 268-274.

19. Landi F, Picca A, Calvani R et al. (2017) Anorexia of aging: assessment and management. Clin Geriatr Med 33, 315-323.

20. Di Francesco V, Barazzoni R, Bissoli L et al. (2010) The quantity of meal fat influences the profile of postprandial hormones as well as hunger sensation in healthy elderly people. J Am Med Dir Assoc 11, 188-193.

21. Morley JE (2003) Anorexia and weight loss in older person. J Gerontol A Biol Sci Med Sci 58, 131-137.

22. Bauer J, Biolo G, Cederholm T et al. (2013) Evidencebased recommendations for optimal dietary protein intake in older people: a position paper from the PROT-AGE Study Group. J Am Med Dir Assoc 14, 542-559.

23. Luca A, Luca M \& Calandra C (2015) Eating Disorders in Late-life. Aging Dis 6, 48-55.
24. Hamerman D (2002) Molecular-based therapeutic approaches in treatment of anorexia of aging and cancer cachexia. J Gerontol A Biol Sci Med Sci 57A, M511-M518.

25. Bodenner D, Spencer T, Riggs AT et al. (2007) A retrospective study of the association between megestrol acetate administration and mortality among nursing home residents with clinically significant weight loss. Am J Geriatr Pharmacother 5, 137-146.

26. Mathey MF, Vanneste VG, de Graaf C et al. (2001) Health effect of improved meal ambiance in a Dutch nursing home: a 1-year intervention study. Prev Med 32, 416-423.

27. Verbrugghe M, Beeckman D, Van Hecke A et al. (2013) Malnutrition and associated factors in nursing home residents: a cross-sectional, multi-centre study. Clin Nutr 32, 438-443.

28. Mir F, Zafar F \& Morley JE (2013) Anorexia of aging: can we decrease protein energy undernutrition in the nursing home? J Am Med Dir Assoc 14, 77-79.

29. Moher D, Liberati A, Tetzlaff J et al., PRISMA Group (2009) Preferred reporting items for systematic reviews and meta-analyses: the PRISMA statement. PLoS Med 6, e1000097.

30. National Library of Medicine, Bethesda, MD, USA. Available at: http://www.pubmed.com (accessed August 2017)

31. Scopus. Available at: http://www.scopus.com (accessed August 2017)

32. Thomson Reuters. Available at: http://www.thomsonreute rs.com (accessed August 2017)

33. Google Scholar. Available at: http://www.scholar.google. com (accessed August 2017).

34. Higgins JP, Altman DG, Gøtzsche PC et al. (2011) The Cochrane Collaboration's tool for assessing risk of bias in randomised trials. BMJ 343, d5928.

35. Thomas P, Hazif-Thomas C \& Clement JP (2003) Influence of antidepressant therapies on weight and appetite in the elderly. JNHA 7, 166-170.

36. Reuben DB, Hirsch SH, Zhou K et al. (2005) The effects of megestrol acetate suspension for elderly patients with reduced appetite after hospitalization. J Am Geriatr Soc 53, 970-975.

37. Pouyssegur V, Brocker P, Schneider SM et al. (2015) An innovative solid oral nutritional supplement to fight weight loss and anorexia: open, randomised controlled trial of efficacy in institutionalised, malnourished older adults. Age Ageing 44, 245-251.

38. Odlund Olin A, Koochek A, Cederholm T et al. (2008) Minimal effect on energy intake by additional evening meal for frail elderly service flat residents - a pilot study. $J$ Nutr Health Aging 12, 295-301.

39. Faxén-Irving G, Andrén-Olsson B, af Geijerstam A et al. (2002) The effect of nutritional intervention in elderly subjects residing in group-living for the demented. Eur $J$ Clin Nutr 56, 221-227. 
40. Wouters-Wesseling W, Wouters AE, Kleijer CN et al. (2002) Study of the effect of a liquid nutrition supplement on the nutritional status of psycho-geriatric nursing home patients. Eur J Clin Nutr 56, 245-251.

41. Chu LW, Lam KS, Tam SC et al. (2001) A randomized controlled trial of low-dose recombinant human growth hormone in the treatment of malnourished elderly medical patients. J Clin Endocrinol Metab 86, 1913-1920.

42. Gazzotti C, Arnaud-Battandier F, Parello M et al. (2003) Prevention of malnutrition in older people during and after hospitalisation: results from a randomised controlled clinical trial. Age Ageing 32, 321-325.

43. Cruz-Jentoft AJ, Calvo JJ, Durán JC et al. (2008) Compliance with an oral hyperproteic supplement with fibre in nursing home residents. J Nutr Health Aging 12, 669-673.

44. Bos C, Benamouzig R, Bruhat A et al. (2000) Short-term protein and energy supplementation activates nitrogen kinetics and accretion in poorly nourished elderly subjects. Am J Clin Nutr 71, 1129-1137.

45. Smoliner C, Norman K, Scheufele R et al. (2008) Effects of food fortification on nutritional and functional status in frail elderly nursing home residents at risk of malnutrition. Nutrition 24, 1139-1144.

46. Lauque S, Arnaud-Battandier F, Mansourian R et al. (2000) Protein-energy oral supplementation in malnourished nursing-home residents. A controlled trial. Age Ageing 29, 51-56.

47. Mathey MF, Siebelink E, de Graaf C et al. (2001) Flavor enhancement of food improves dietary intake and nutritional status of elderly nursing home residents. $J$ Gerontol A Biol Sci Med Sci 56, M200-M205.

48. Essed NH, van Staveren WA, Kok FJ et al. (2007) No effect of 16 weeks flavor enhancement on dietary intake and nutritional status of nursing home elderly. Appetite $\mathbf{4 8}$, 29-36.

49. Appleton KM (2009) Increases in energy, protein and fat intake following the addition of sauce to an older person's meal. Appetite 52, 161-165.

50. Nijs KA, de Graaf C, Siebelink E et al. (2006) Effect of family-style meals on energy intake and risk of malnutrition in dutch nursing home residents: a randomized controlled trial. J Gerontol A Biol Sci Med Sci 61, 935-942.

51. Lou MF, Dai YT, Huang GS et al. (2007) Nutritional status and health outcomes for older people with dementia living in institutions. J Adv Nurs 60, 470-477.

52. Simmons SF, Keeler E, Zhuo X et al. (2008) Prevention of unintentional weight loss in nursing home residents: a controlled trial of feeding assistance. J Am Geriatr Soc 56, 1466-1473.

53. de Jong N, Chin A, Paw MJ et al. (1999) Functional biochemical and nutrient indices in frail elderly people are partly affected by dietary supplements but not by exercise. J Nutr 129, 2028-2036.
54. Malafarina V, Uriz-Otano F, Gil-Guerrero L et al. (2013) The anorexia of ageing: physiopathology, prevalence, associated comorbidity and mortality. A systematic review. Maturitas 74, 293-302.

55. Morley JE (2013) Pathophysiology of the anorexia of aging. Curr Opin Clin Nutr Metab Care 16, 27-32.

56. Morley JE, Arai H, Cao L et al. (2017) Integrated care: enhancing the role of the primary health care professional in preventing functional decline: a systematic review. J Am Med Dir Assoc 18, 489-494.

57. Hilas O \& Avena-Woods C (2014) Potential role of mirtazapine in underweight older adults. Consult Pharm 29, 124-130.

58. Volkert D \& Schrader E (2013) Dietary assessment methods for older persons: what is the best approach? Curr Opin Clin Nutr Metab Care 16, 534-540.

59. Kmieć Z, Pétervári E, Balaskó M et al. (2013) Anorexia of aging. Vitam Horm 92, 319-355.

60. Christner S, Ritt M, Volkert D et al. (2016) Evaluation of the nutritional status of older hospitalised geriatric patients: a comparative analysis of a Mini Nutritional Assessment (MNA) version and the Nutritional Risk Screening (NRS 2002). J Hum Nutr Diet 29, 704713.

61. Cooper PL, Raja R, Golder J et al. (2016) Implementation of nutrition risk screening using the Malnutrition Universal Screening Tool across a large metropolitan health service. J Hum Nutr Diet 29, 697-703.

62. Smith S \& Madden AM (2016) Body composition and functional assessment of nutritional status in adults: a narrative review of imaging, impedance, strength and functional techniques. J Hum Nutr Diet 29, 714732.

63. Jiang SM, Jia L, Liu J et al. (2016) Beneficial effects of antidepressant mirtazapine in functional dyspepsia patients with weight loss. World J Gastroenterol 22, 5260-5266.

64. Rigler SK, Webb MJ, Redford L et al. (2001) Weight outcomes among antidepressant users in nursing facilities. J Am Geriatr Soc 49, 49-55.

65. Abizanda P, López MD, García VP et al. (2015) Effects of an oral nutritional supplementation plus physical exercise intervention on the physical function, nutritional status, and quality of life in frail institutionalized older adults: the ACTIVNES Study. J Am Med Dir Assoc 16, 439.e9-439.e16.

66. Rondanelli M, Opizzi A, Antoniello N et al. (2011) Effect of essential amino acid supplementation on quality of life, amino acid profile and strength in institutionalized elderly patients. Clin Nutr 30, 571-577.

67. Trabal J \& Farran-Codina A (2015) Effe Effects of dietary enrichment with conventional foods on energy and protein intake in older adults: a systematic review. Nutr Rev 73, 624-633.

68. Yeomans MR, Gray RW \& Conyers TH (1998) Maltodextrin preloads reduce food intake without altering the appetiser effect. Physiol Behav 64, 501-506. 
69. Wilson MM, Purushothaman R \& Morley JE (2002) Effect of liquid dietary supplements on energy intake in the elderly. Am J Clin Nutr 75, 944-947.

70. Somekawa S, Mine T, Ono K et al. (2017) Relationship between sensory perception and frailty in a communitydwelling elderly population. J Nutr Health Aging 21, 710-714.

71. Syed Q, Hendler KT \& Koncilja K (2016) The impact of aging and medical status on dysgeusia. Am J Med 129, 753.e1-6.

72. Havermans RC, Geschwind N, Filla S et al. (2009) Sensory-specific satiety is unaffected by manipulations of flavour intensity. Physiol Behav 97, 327-333.

73. Prescott J (2004) Effects of added glutamate on liking for novel food flavors. Appetite 42, 143-150.

74. Nagraj SK, Naresh S, Srinivas, K et al. (2014) Interventions for the management of taste disturbances. Cochrane Database Syst Rev, 12, CD010470.
75. Essed NH, Oerlemans P, Hoek M et al. (2009) Optimal preferred MSG concentration in potatoes, spinach and beef and their effect on intake in institutionalized elderly people. J Nutr Health Aging 13, 769-775.

76. Richard D (1995) Exercise and the neurobiological control of food intake and energy expenditure. Int J Obesity 19, S73-S79.

\section{Supporting information}

Additional supporting information may be found online in the Supporting Information section at the end of the article.

Table S1. General characteristics of the studies considered.

Table S2. Therapeutic approach and results obtained in the studies considered. 